\title{
SUMMATION IDENTITIES FOR REPRESENTATION OF CERTAIN REAL NUMBERS
}

\author{
GEORGE GROSSMAN, AKALU TEFERA, AND AKLILU ZELEKE
}

Received 27 March 2006; Accepted 28 March 2006

We present identities used to represent real numbers of the form $x u^{m} \pm y v^{n}$ for appropriately chosen real numbers $x, y, u, v$ and nonnegative integers $m$ and $n$. We present the proofs of the identities by applying Zeilberger's algorithm.

Copyright (c) 2006 Hindawi Publishing Corporation. All rights reserved.

\section{Introduction}

It is well known that every integer can be written as a sum of integral powers of 2 . A somewhat related problem is to find for every positive integer $n$ a positive integer $k$ depending on $n$ with $k(n)<k(n+1)$ and integer coefficients $a_{i}, i=1,2, \ldots, k-1$, such that

$$
n=\sum_{i=0}^{k-1} a_{i} 2^{2 i} .
$$

The background and motivation for studying this problem lies in finding the zeros of the $j$ th-order generalized Fibonacci polynomial defined by $F_{j}(x)=x^{j-1}-\cdots-x-1$. Dubeau [1] and Flores [3] have shown that the positive roots of $F_{j}(x)$ are of the form $2-O\left(2^{-j}\right)$. Grossman and Narayan [4] have proven that the single negative zero of $F_{j}(x)$ has the form $-1+O\left(j^{-1}\right)$ for $j$ even and tends to -1 monotonically as $j \rightarrow \infty$. Using the fact that

$$
F_{j}(x)=\left(x-2+\varepsilon_{j}\right)\left(x+1-\delta_{j}\right)\left(x^{j-2}+a_{j-3} x^{j-3}+\cdots+a_{1} x+a_{0}\right),
$$

where $\delta_{j}$ and $\varepsilon_{j}$ are positive, decreasing sequences for $j=4,6, \ldots$, Grossman and Zeleke [6] have found an explicit form for the coefficients $a_{i}$, where $i=0,1, \ldots, j-3$, in terms of $\delta$ and $\varepsilon$. The solution was found by solving a nonhomogeneous linear recurrence relation of the form $-a_{n}+b a_{n+1}+c a_{n+2}=1$, where $b=\varepsilon-1-\delta, c=(1-\delta)(2-\varepsilon)$. As byproduct of this solution, several combinatorial identities were formulated by considering evenand odd-indexed terms of $a_{n}$. 
Some of these identities give representations of positive integers using binomial coefficients. Others are identities for representing certain types of real numbers. In this paper, we call these identities "real number identities." Real number identities are interesting for various reasons. On one side, formulation and proofs of combinatorial identities traditionally have been linked to arguments that have their origin in counting problems. Such identities can then be used to represent positive integers. From this perspective, it is not obvious that one can formulate real number identities. This by itself makes the attempt to represent real numbers in terms of combinatorial identities interesting. Furthermore, by considering some special cases, we can use the real number identities to generate interesting sequences. We give five examples to demonstrate this fact. In the first example, we consider a representation of the integer sequence $2^{n}, n \geq 0$. What is different about this representation is that the summand in the identity involves irrational terms. In example two, we discuss an alternate way to generate the Fibonacci sequence $F_{n}$ with initial values $F_{1}=F_{2}=1$. The third example shows how to generate a shifted even-indexed Fibonacci sequence $F_{2 n+2}, n \geq 1$. The fourth example shows how to generate from Theorem 2.1 integer solutions of a Pell equation of the form $x^{2}-3 y^{2}=1$. In example five, we give an identity that can be used to represent a certain type of rational numbers.

The real number identities in this paper have been introduced in [6] where the methods of proofs involve induction, previously known combinatorial identities, and algebraic manipulations. The goal of this paper is to present computer-generated proofs using Zeilberger's algorithm. For a superb exposition of Zeilberger's algorithm see, among others, the books $A=B$ [8] and Hypergeometric Summation [7] which are devoted to this and other methods. The paper is organized as follows. In Section 2 we present several real number identities. Computer-generated proofs of these identities are presented in Section 3. Throughout this paper, we denote the set $\{k, k+1, k+2, \ldots\}$ for $k \in \mathbb{Z}$ by $\mathbb{N}_{k}$.

\section{Main theorems}

Theorem 2.1. For $n \in \mathbb{N}_{0}, b \in \mathbb{R} \backslash\{-2,-1\}$,

$$
\frac{1}{(b+1)^{n+2}} \sum_{k=0}^{n} \frac{b^{2 k+1}}{(b+1)^{k}}\left(\begin{array}{c}
n+k+1 \\
2 k+1
\end{array}\right)=\frac{1}{b+2}\left(1-\frac{1}{(b+1)^{2(n+1)}}\right) .
$$

Theorem 2.2. Let $n \in \mathbb{N}_{1}$ and let $b, c \in \mathbb{R} \backslash\{0\}$ such that $b^{2}+4 c>0$. Then

$$
\sum_{k=1}^{n} \frac{1}{2^{n} b^{2}}\left(\frac{b}{2 c}\right)^{2 n}\left(1+\frac{4 c}{b^{2}}\right)^{k-1}\left(\begin{array}{c}
2 n \\
2 k-1
\end{array}\right)=\frac{\alpha \beta}{\alpha-\beta}\left(\alpha^{n}-\beta^{n}\right),
$$

where $\alpha=1 /\left(b^{2}+2 c-\sqrt{b^{2}\left(b^{2}+4 c\right)}\right)$ and $\beta=1 /\left(b^{2}+2 c+\sqrt{b^{2}\left(b^{2}+4 c\right)}\right)$.

Theorem 2.3. For $n \in \mathbb{N}_{-1}, b \in \mathbb{R} \backslash\{-2,-1\}$,

$$
\sum_{k=0}^{n+1} \frac{b^{2 k}}{(b+1)^{n+k+1}}\left(\begin{array}{c}
n+k+1 \\
2 k
\end{array}\right)=\frac{b+1}{b+2}\left(1+\frac{1}{(b+1)^{2 n+3}}\right) .
$$


Theorem 2.4. Let $n \in \mathbb{N}_{1}$ and let $b, c \in \mathbb{R}$ such that $b \neq 0$ and $c>0$. Then

$$
\sum_{k=0}^{n}\left(\frac{b}{2^{n-1}}\right)\left(\frac{b}{2 c}\right)^{2 n+1}\left(1+\frac{4 c}{b^{2}}\right)^{k+1}\left(\begin{array}{l}
2 n+1 \\
2 k+1
\end{array}\right)=\frac{\sqrt{\alpha}+\sqrt{\beta}}{\sqrt{\alpha \beta}}\left(\beta^{n+1 / 2}-\alpha^{n+1 / 2}\right),
$$

where $\alpha=1 /\left(b^{2}+2 c-\sqrt{b^{2}\left(b^{2}+4 c\right)}\right)$ and $\beta=1 /\left(b^{2}+2 c+\sqrt{b^{2}\left(b^{2}+4 c\right)}\right)$.

Theorem 2.5. For $n \in \mathbb{N}_{1}, b \in \mathbb{R}\{-2,-1\}$,

$$
\sum_{k=0}^{n-1} \sum_{m=2 k+2}^{n+1+k} \frac{b^{m}}{(b+1)^{n+k+1}}\left(\begin{array}{c}
n+k+1 \\
m
\end{array}\right)=\frac{b(n+1)}{b+2}-\frac{(b+1)^{2}}{(b+2)^{2}}\left(1-\frac{1}{(b+1)^{2 n+2}}\right) .
$$

Remark 2.6. By substituting specific values for the parameters in the above theorems, one can get interesting identities. We consider five examples.

Example 2.7 (identities for $2^{n}$ ). By substituting $b=-1+\sqrt{2} / 2$ in Theorem 2.1 , we get the following identity for the integer sequence $2^{n}$ :

$$
\frac{1}{2}+\frac{2^{n / 2}}{2} \sum_{k=0}^{n}\left(\frac{3}{\sqrt{2}}-2\right)^{k}\left(\begin{array}{c}
n+k+1 \\
2 k+1
\end{array}\right)=2^{n}
$$

If we let $b=-1-\sqrt{2} / 2$, we get a different identity for the sequence $2^{n}$ :

$$
\frac{1}{2}+\frac{2^{n / 2}}{2} \sum_{k=0}^{n}(-1)^{n+k}\left(\frac{3}{\sqrt{2}}+2\right)^{k}\left(\begin{array}{c}
n+k+1 \\
2 k+1
\end{array}\right)=2^{n}
$$

These two identities are different from other representations of $2^{n}$ in the sense that they involve irrational terms to generate an integer sequence. They are also interesting from this perspective.

Example 2.8 (generating the Fibonacci sequence.). In Theorem 2.2, choose $c= \pm 1 / 2$ and $b= \pm \sqrt{(\sqrt{5}-2) / 2}$. This makes $\alpha=(\sqrt{5}+1) / 2$ and $\beta=(\sqrt{5}-1) / 2$. Note that since $\alpha \beta=$ $\alpha-\beta=1$, the right-hand side of Theorem 2.2 is the sequence

$$
G_{n}=\left(\frac{\sqrt{5}+1}{2}\right)^{n}-\left(\frac{\sqrt{5}-1}{2}\right)^{n}, \quad n \geq 1
$$

By setting $F_{0}=G_{0}=0$, we get the following relation between $G_{n}$ and $F_{n}$ for $n \geq 0$ :

$$
G_{2 n}=\sqrt{5} F_{2 n}, \quad G_{2 n+1}=F_{2 n}+F_{2 n+2}
$$

From this relation, we see that Theorem 2.2 provides a combinatorial identity to generate the Fibonacci sequence. 


\section{Summation identities for real numbers representation}

Example 2.9 (generating a shifted even-indexed Fibonacci sequence). In Theorem 2.1, if we let $b=(1 \pm \sqrt{5}) / 2$, then $b^{2} /(b+1)=1$ and Theorem 2.1 takes the form

$$
\sum_{k=0}^{n}\left(\begin{array}{c}
n+k+1 \\
2 k+1
\end{array}\right)=\left(\frac{5+3 \sqrt{5}}{10}\right)\left(\frac{3+\sqrt{5}}{2}\right)^{n}+\left(\frac{5-3 \sqrt{5}}{10}\right)\left(\frac{3-\sqrt{5}}{2}\right)^{n} .
$$

One can show that the right-hand side of $(2.10)$ is $F_{2 n+2}$.

Example 2.10. By substituting $b=1 \pm \sqrt{3}$ in Theorem 2.1, we get the following identity that generates the sequence $a_{0}=1, a_{1}=4$, and $a_{n+2}=4 a_{n+1}-a_{n}$ for $n \geq 0$ :

$$
\sum_{k=0}^{n} 2^{k}\left(\begin{array}{c}
n+k+1 \\
2 k+1
\end{array}\right)=\left(\frac{1}{2}+\frac{\sqrt{3}}{3}\right)(2+\sqrt{3})^{n}+\left(\frac{1}{2}-\frac{\sqrt{3}}{3}\right)(2-\sqrt{3})^{n} .
$$

The first few terms of the sequence $a_{n}$ are $1,4,15,56,209,780, \ldots$ One interesting property of these terms is that $3 a_{n}{ }^{2}+1$ is a perfect square.

Example 2.11. By substituting $b=1$ in Theorem 2.1 and $b=-1, c=2$ in Theorem 2.2, we get the identities discovered in [6] for real numbers of the form $(1 / 6)\left(1-1 / 4^{n}\right)$.

\section{Proofs of the theorems}

Proof of Theorem 2.1. Since $\left(\begin{array}{c}n+k+1 \\ 2 k+1\end{array}\right)=0$ for $k>n+1$ and $k<0$, the identity in Theorem 2.1 can be rewritten as

$$
\frac{1}{(b+1)^{n+2}} \sum_{k \in \mathbb{Z}} \frac{b^{2 k+1}}{(b+1)^{k}}\left(\begin{array}{c}
n+k+1 \\
2 k+1
\end{array}\right)=\frac{1}{b+2}\left(1-\frac{1}{(b+1)^{2(n+1)}}\right) .
$$

This is also equivalent to

$$
\sum_{k \in \mathbb{Z}} \frac{(2+b) b^{2 k+1}}{(b+1)^{k-n}}\left(\begin{array}{c}
n+k+1 \\
2 k+1
\end{array}\right)=(1+b)^{2(n+1)}-1 .
$$

Let us denote the left-hand side of $(3.2)$ by $S_{b}(n)$. Let $F_{b}(n, k)$ denote the summand of $S_{b}(n)$, that is,

$$
F_{b}(n, k)=\frac{(2+b) b^{2 k+1}}{(b+1)^{k-n}}\left(\begin{array}{c}
n+k+1 \\
2 k+1
\end{array}\right)
$$

Then $F_{b}$ satisfies the recurrence equation (the recurrence equation is automatically generated by the Maple package ekhad which is available (for free) from http://www.math .rutgers.edu/ ${ }^{\sim}$ zeilberg/):

$$
\begin{gathered}
-(b+1)^{2} F_{b}(n, k)+\left(b^{2}+2 b+2\right) F_{b}(n+1, k)-F_{b}(n+2, k)=G_{b}(n, k+1)-G_{b}(n, k), \\
\text { where } G_{b}(n, k)=(2+b) b^{2 k+1}(b+1)^{n-k+2}\left(\begin{array}{c}
n+k+1 \\
2 k-1
\end{array}\right) .
\end{gathered}
$$


By summing both sides of (3.4) with respect to $k$, we get

$$
-(b+1)^{2} S_{b}(n)+\left(b^{2}+2 b+2\right) S_{b}(n+1)-S_{b}(n+2)=0 .
$$

Moreover, $S_{b}(0)=(b+1)^{2}-1$ and $S_{b}(1)=(1+b)^{4}-1$. Also $(1+b)^{2(n+1)}-1$ satisfies (3.5), and hence $S_{b}(n)=(1+b)^{2(n+1)}-1$.

Remarks 3.1. (1) In the sequel, we will use the same proof style. Compute a recurrence relation of order $d$ for the summand and sum the summand recurrence with respect to the summation index to get a recurrence for the sum $S(n)$. Check that the right-hand side also satisfies the same recurrence and that it agrees with $S(n)$ for the first $d$ initial values of $n$. Then the identity follows.

(2) We would like to note that one can also get the right-hand side of (3.2) by directly solving the recurrence (3.5) which has constant coefficients. For more details, see for instance $[8,9]$.

To make the proofs more compact, we introduce the following operator notations.

Defintion 3.2. Given a function $F(n, k)$ of the (discrete) variables $n$ and $k$, define the forward shift operator $E_{n}$ by $E_{n} F(n, k)=F(n+1, k)$.

With this notation, (3.4) becomes

$$
\left(-(b+1)^{2}+\left(b^{2}+2 b+2\right) E_{n}-E_{n}^{2}\right) F_{b}(n, k)=G_{b}(n, k+1)-G_{b}(n, k) .
$$

From the proof of Theorem 2.1, we observe that to certify the truth of the identity, it would suffice to display the pair $\left(P, G_{b}\right)$ (called a " $Z$-pair"), where $P=-(b+1)^{2}+\left(b^{2}+\right.$ $2 b+2) E_{n}-E_{n}^{2}$.

In the sequel for a given function $F(n, k)$, a $Z$-pair $(P, G)$ means that $P F(n, k)=G(n$, $k+1)-G(n, k)$. Since the $Z$-pair actually certifies the truth of the identity, it is called the proof certificate. Therefore, in the proofs of Theorems 2.2, 2.4, we only give the proof certificates.

Proof of Theorem 2.2. Since $\left(\begin{array}{c}2 n \\ 2 k-1\end{array}\right)=0$ for $k>n$ and $k<1$, the identity in Theorem 2.2 can be rewritten as

$$
\sum_{k \in \mathbb{Z}} \frac{1}{2^{n} b^{2}}\left(\frac{b}{2 c}\right)^{2 n}\left(1+\frac{4 c}{b^{2}}\right)^{k-1}\left(\begin{array}{c}
2 n \\
2 k-1
\end{array}\right)=\frac{\alpha^{n}-\beta^{n}}{\alpha \beta(\alpha-\beta)} .
$$

Let us denote the summand of the left-hand side of (3.7) by $F_{b, c}(n, k)$, that is,

$$
F_{b, c}(n, k)=\frac{1}{2^{n} b^{2}}\left(\frac{b}{2 c}\right)^{2 n}\left(1+\frac{4 c}{b^{2}}\right)^{k-1}\left(\begin{array}{c}
2 n \\
2 k-1
\end{array}\right) .
$$


6 Summation identities for real numbers representation

The required $Z$-pair $\left(P, G_{b, c}\right)$ is $P=1-\left(2 b^{2}+4 c\right) E_{n}+4 c^{2} E_{n}{ }^{2}$ and

$$
G_{b, c}(n, k)=\left(\frac{1}{2^{n+1} b^{2}}\right)\left(\frac{b}{2 c}\right)^{2 n+2}\left(1+\frac{4 c}{b^{2}}\right)^{k-1}\left(-\frac{(3 n-2 k+6) b^{2}}{2 k-3}\left(\begin{array}{c}
2 n+1 \\
2 k-4
\end{array}\right)+2 c\left(\begin{array}{c}
2 n \\
2 k-3
\end{array}\right)\right) .
$$

Proof of Theorem 2.3. Since $\left(\begin{array}{c}n+k+1 \\ 2 k\end{array}\right)=0$ for $k>n+1$ and $k<0$, the identity in Theorem 2.3 can be rewritten as

$$
\sum_{k \in \mathbb{Z}} \frac{b^{2 k}}{(b+1)^{n+k+1}}\left(\begin{array}{c}
n+k+1 \\
2 k
\end{array}\right)=\frac{b+1}{b+2}\left(1+\frac{1}{(b+1)^{2 n+3}}\right) .
$$

The $Z$-pair $\left(P, G_{b}\right)$ associated with the summand $F_{b}(n, k)$ of the left-hand side of $(3.10)$ is

$$
P=1-\left(b^{2}+2 b+2\right) E_{n}+(b+1)^{2} E_{n}{ }^{2}, \quad G_{b}(n, k)=-\frac{b^{2 k}}{(b+1)^{n+k+1}}\left(\begin{array}{c}
n+k+1 \\
2 k-2
\end{array}\right) .
$$

Proof of Theorem 2.4. Since $\left(\begin{array}{c}2 n+1 \\ 2 k+1\end{array}\right)=0$ for $k>n$ and $k<0$, the identity in Theorem 2.4 can be rewritten as

$$
\sum_{k \in \mathbb{Z}}\left(\frac{b}{2^{n-1}}\right)\left(\frac{b}{2 c}\right)^{2 n+1}\left(1+\frac{4 c}{b^{2}}\right)^{k+1}\left(\begin{array}{l}
2 n+1 \\
2 k+1
\end{array}\right)=\frac{\sqrt{\alpha}+\sqrt{\beta}}{\sqrt{\alpha \beta}}\left(\beta^{n+1 / 2}-\alpha^{n+1 / 2}\right) .
$$

The $Z$-pair $\left(P, G_{b, c}\right)$ associated with the summand $F_{b, c}(n, k)$ of the left-hand side of (3.12) is

$$
\begin{gathered}
P=1-\left(2 b^{2}+4 c\right) E_{n}+4 c^{2} E_{n}{ }^{2} \\
G_{b, c}(n, k)=\left(\frac{b}{2^{n-1}}\right)\left(\frac{b}{2 c}\right)^{2 n+3}\left(1+\frac{4 c}{b^{2}}\right)^{k+1}\left(-\frac{(6 n-4 k+11) b^{2}}{8 k-4}\left(\begin{array}{l}
2 n+2 \\
2 k-2
\end{array}\right)+\left(\begin{array}{l}
2 n+1 \\
2 k-1
\end{array}\right) c\right) .
\end{gathered}
$$

Proof of Theorem 2.5. Reversing the order of summation, the identity can be rewritten as

$$
\sum_{m=2}^{2 n} \sum_{k=0}^{\lfloor(m-2) / 2\rfloor} \frac{b^{m}}{(b+1)^{n+k+1}}\left(\begin{array}{c}
n+k+1 \\
m
\end{array}\right)=\frac{b(n+1)}{b+2}-\frac{(b+1)^{2}}{(b+2)^{2}}\left(1-\frac{1}{(b+1)^{2 n+2}}\right) .
$$

Let us denote the left-hand side of (3.14) by $S_{b}(n)$ and its summand by $F_{b}(n, m, k)$, that is,

$$
F_{b}(n, m, k)=\frac{b^{m}}{(b+1)^{n+k+1}}\left(\begin{array}{c}
n+k+1 \\
m
\end{array}\right) .
$$


Then $F_{b}(n, m, k)=F_{b}(k, m, n)$ and $F_{b}(n+1, m, k)=F_{b}(n, m, k+1)$. This implies that

$$
F_{b}(n+1, m, k)-F_{b}(n, m, k)=F_{b}(n, m, k+1)-F_{b}(n, m, k) .
$$

Summing both sides of (3.16) with respect to $k$ and with respect to $m$, we get

$$
\begin{aligned}
& S_{b}(n+1)-\frac{b^{2 n+1}}{(b+1)^{2 n+1}}-\frac{b^{2 n+2}}{(b+1)^{2 n+2}}-S_{b}(n) \\
& \quad=\sum_{m=2}^{2 n}\left(\begin{array}{c}
n+\left\lfloor\frac{m}{2}\right\rfloor+1 \\
m
\end{array}\right) \frac{b^{m}}{(b+1)^{n+\lfloor m / 2\rfloor+1}}-\sum_{m=2}^{2 n}\left(\begin{array}{c}
n+1 \\
m
\end{array}\right) \frac{b^{m}}{(b+1)^{n+1}} .
\end{aligned}
$$

But

$$
\begin{aligned}
\sum_{m=2}^{2 n}\left(\begin{array}{c}
n+1 \\
m
\end{array}\right) \frac{b^{m}}{(b+1)^{n+1}}=\frac{1}{(b+1)^{n+1}}\left(\sum_{m=0}^{n+1}\left(\begin{array}{c}
n+1 \\
m
\end{array}\right) b^{m}-1-(n+1) b\right) & =\frac{1}{(b+1)^{n+1}}\left((b+1)^{n+1}-1-(n+1) b\right) \\
= & 1-\frac{1}{(b+1)^{n+1}}-\frac{(n+1) b}{(b+1)^{n+1}}, \\
\sum_{m=2}^{2 n}\left(\begin{array}{c}
n+\left\lfloor\frac{m}{2}\right\rfloor+1 \\
m
\end{array}\right) \frac{b^{m}}{(b+1)^{n+\lfloor m / 2\rfloor+1}} & \sum_{m=1}^{n}\left(\begin{array}{c}
n+m+1 \\
2 m
\end{array}\right) \frac{b^{2 m}}{(b+1)^{n+m+1}}+\sum_{m=1}^{n-1}\left(\begin{array}{c}
n+m+1 \\
2 m+1
\end{array}\right) \frac{b^{2 m+1}}{(b+1)^{n+m+1}} \\
= & \sum_{m=0}^{n+1}\left(\begin{array}{c}
n+m+1 \\
2 m
\end{array}\right) \frac{b^{2 m}}{(b+1)^{n+m+1}}-\frac{1}{(b+1)^{n+1}}-\frac{b^{2 n+2}}{(b+1)^{2 n+2}} \\
& +\sum_{m=0}^{n}\left(\begin{array}{c}
n+m+1 \\
2 m+1
\end{array}\right) \frac{b^{2 m+1}}{(b+1)^{n+m+1}}-\frac{(n+1) b}{(b+1)^{n+1}}-\frac{b^{2 n+1}}{(b+1)^{2 n+1}} \\
= & \frac{b+1}{b+2}\left(1+\frac{1}{(b+1)^{2 n+3}}\right)-\frac{1}{(b+1)^{n+1}}-\frac{b^{2 n+2}}{(b+1)^{2 n+2}} \\
& +\frac{b+1}{b+2}\left(1-\frac{1}{(b+1)^{2 n+2}}\right)-\frac{(n+1) b}{(b+1)^{n+1}}-\frac{b^{2 n+1}}{(b+1)^{2 n+1}} .
\end{aligned}
$$

From (3.17) and (3.18), we get that

$$
S_{b}(n+1)-S_{b}(n)=\frac{b}{b+2}\left(1-\frac{1}{(b+2)^{2 n+2}}\right)
$$


and $S_{b}(1)=b^{2} /(b+1)^{2}$. The right-hand side of (3.14) also satisfies this recurrence relation. Hence

$$
S_{b}(n)=\frac{b(n+1)}{b+2}-\frac{(b+1)^{2}}{(b+2)^{2}}\left(1-\frac{1}{(b+1)^{2 n+2}}\right) \quad \forall n \in \mathbb{N}
$$

Remark 3.3. We would like to mention that the recurrence (3.16) can also be automatically generated by MultiSum [10], a Mathematica package which is available from the website http://www.risc.uni-linz.ac.at/research/combinat/software/.

\section{Acknowledgment}

The authors are grateful for helpful suggestions and valuable comments from the referee.

\section{References}

[1] F. Dubeau, On r-generalized Fibonacci numbers, The Fibonacci Quarterly 27 (1989), no. 3, 221229.

[2] EKHAD, a MAPLE package by Doron Zeilbeger, http://www.math.rutgers.edu/ zeilberg/.

[3] I. Flores, Direct calculation of k-generalized Fibonacci numbers, The Fibonacci Quarterly 5 (1967), no. 3, 259-266.

[4] G. Grossman and S. Narayan, On the characteristic polynomials of the jth order Fibonacci sequence, Applications of Fibonacci Numbers, Vol. 8 (Rochester, NY, 1998) (F. T. Howard, ed.), Kluwer Academic, Dordrecht, 1999, pp. 165-177.

[5] G. Grossman, A. Tefera, and A. Zeleke, On proofs of certain combinatorial identities, to appear in Proceedings of the 11th International Conference on Fibonacci Numbers and Their Applications.

[6] G. Grossman and A. Zeleke, On linear recurrence relations, Journal of Concrete and Applicable Mathematics 1 (2003), no. 3, 229-245.

[7] W. Koepf, Hypergeometric Summation: An Algorithmic Approach to Summation and Special Function Identities, American Mathematical Society, Rhode Island, 1998.

[8] M. Petkovšek, H. S. Wilf, and D. Zeilberger, $A=B$, A. K. Peters, Massachusetts, 1996.

[9] R. P. Stanley, Enumerative Combinatorics. Vol. 1, Cambridge Studies in Advanced Mathematics, vol. 49, Cambridge University Press, Cambridge, 1997.

[10] K. Wegschaider, Computer generated proofs of binomial multi-sum identities, Diploma thesis, RISC, J. Kepler University, Linz, 1997.

George Grossman: Department of Mathematics, Central Michigan University,

Mount Pleasant, MI 48859, USA

E-mail address: gross1gw@cmich.edu

Akalu Tefera: Department of Mathematics, Grand Valley State University, Allendale,

MI 49401, USA

E-mail address: teferaa@gvsu.edu

Aklilu Zeleke: Lyman Briggs School, Michigan State University, East Lansing, MI 48825-1107, USA; Department of Statistics and Probability, Michigan State University, East Lansing, MI 48824-1027, USA

E-mail address: zeleke@stt.msu.edu 


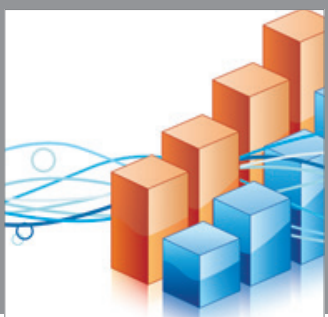

Advances in

Operations Research

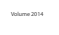

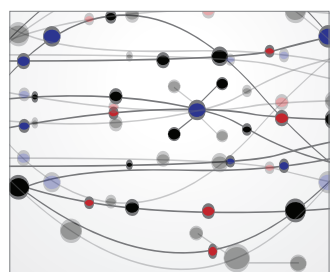

\section{The Scientific} World Journal
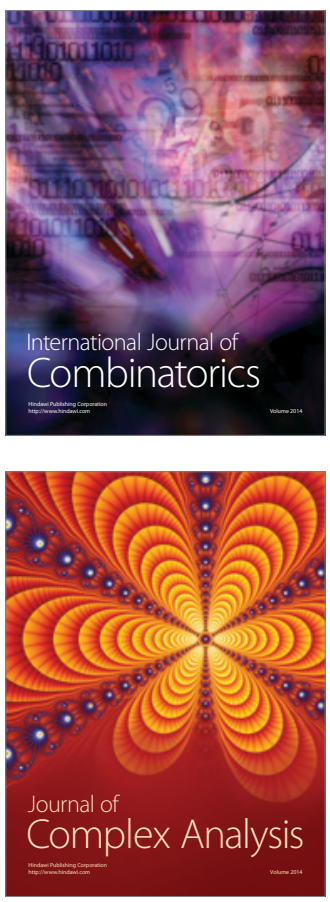

International Journal of

Mathematics and

Mathematical

Sciences
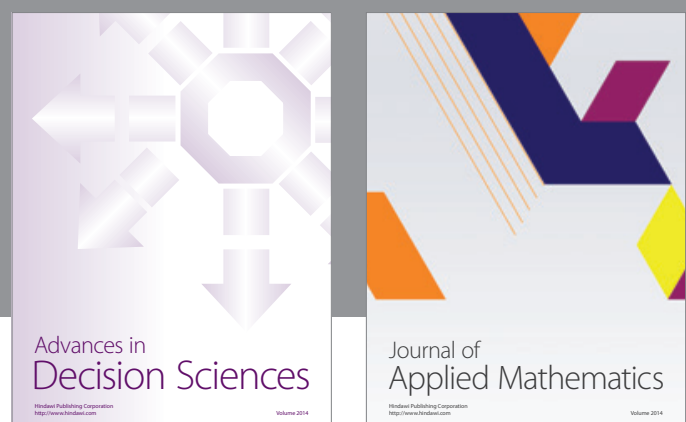

Journal of

Applied Mathematics
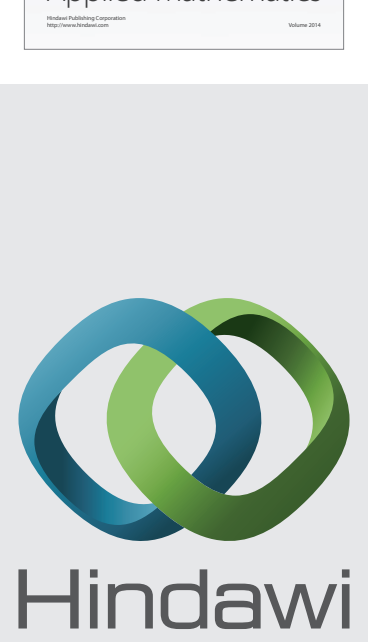

Submit your manuscripts at http://www.hindawi.com
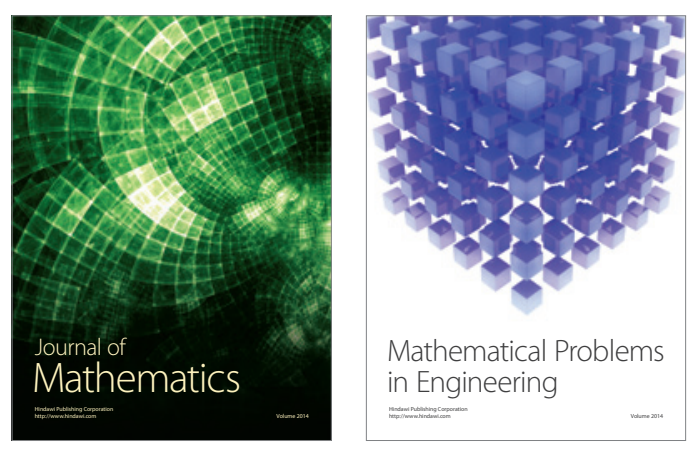

Mathematical Problems in Engineering
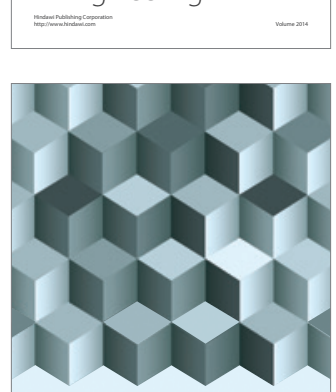

Journal of

Function Spaces
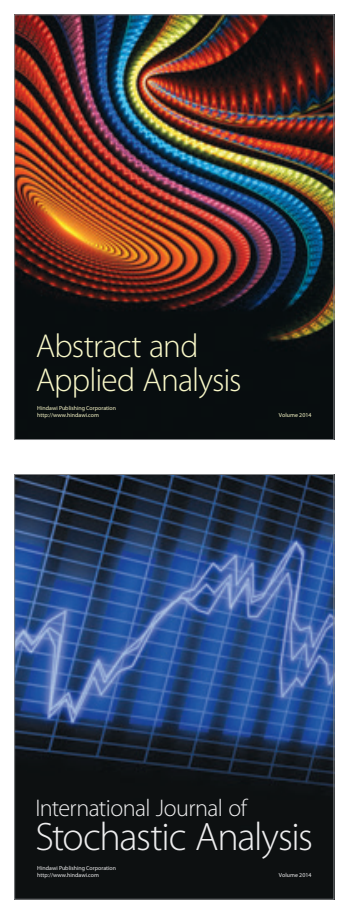

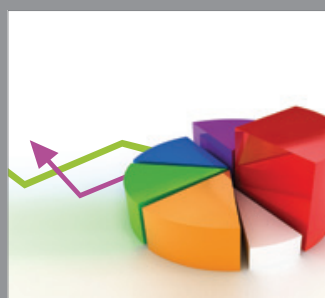

ournal of

Probability and Statistics

Promensencen
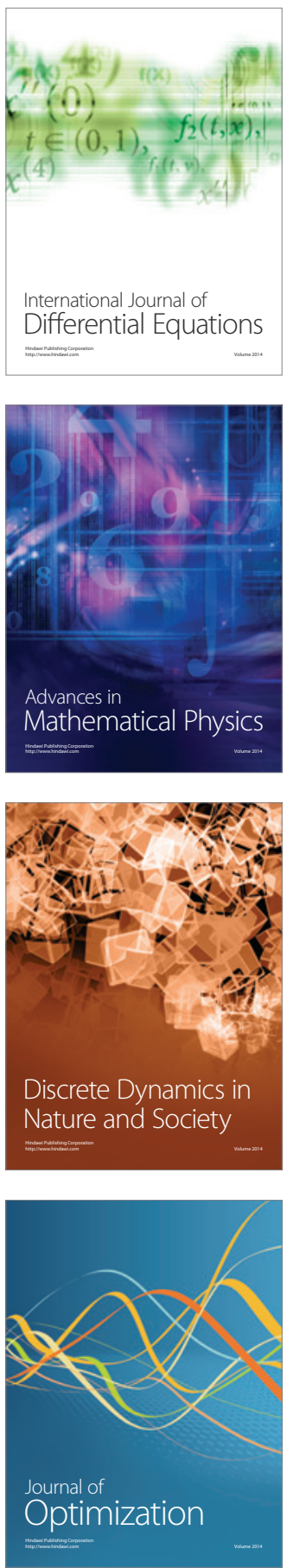